\title{
DADOS EPIDEMIOLÓGICOS DA LITÍASE RENAL, EM HOSPITAL DE REFERÊNCIA, EM BELO HORIZONTE, MINAS GERAIS
}

\author{
EPIDEMIOLOGY OF RENAL CALCULI IN A REFERENCE HOSPITAL \\ IN BELO HORIZONTE, MINAS GERAIS STATE
}

Andy Petroianu ${ }^{1}$; José Estevão de Oliveira Neto² \& Luiz R Alberti ${ }^{3}$

\begin{abstract}
${ }^{1}$ Professor Titular do Departamento de Cirurgia da Faculdade de Medicina - UFMG, Docente Livre da Faculdade de Medicina de Ribeirão Preto - USP e da Escola Paulista de Medicina - UNIFESP, Doutor em Fisiologia e Farmacologia, Pesquisador IA do CNPq. ${ }^{2}$ Médico. ${ }^{3}$ Acadêmico de Medicina da Faculdade de Ciências Médicas de Minas Gerais. Instituição:Departamento de Cirurgia, Faculdade de Medicina - UFMG. Av. Alfredo Balena, n 190, Belo Horizonte, MG, 30130-100

Correspondência: Prof. Andy Petroianu. Av. Afonso Pena, n 1626, Apto 1901. 30130-005, Belo Horizonte, MG. FAX: (31) 3274-7744
\end{abstract}

PETROIANU A; OLIVEIRA NETO JE \& ALBERTI LR. Dados epidemiológicos da litíase renal, em hospital de referência, em Belo Horizonte, Minas Gerais. Medicina, Ribeirão Preto, 34: 85-88, jan./mar. 2001.

RESUMO: Introdução: A litíase renal é uma afecção muito comum na prática clínica e múltiplos fatores estão relacionados com sua etiopatogenia, embora não tenhamos encontrado estudo algum sobre a epidemiologia da litíase no Brasil.

Objetivo: Avaliar a influência da idade, do sexo, da cor da pele e da lateralidade como fatores de risco para cálculo renal.

Pacientes e Métodos: Foram estudados 400 prontuários de pacientes com diagnóstico de litíase urinária, nos Serviços de Nefrologia e Urologia do Hospital das Clínicas da Universidade Federal de Minas Gerais, em Belo Horizonte. Todos os diagnósticos foram confirmados pela história clínica associada a métodos de imagem.

Resultados: Houve predomínio de pacientes do sexo feminino $(54,5 \%)$ sobre o masculino com litíase renal. Os doentes brancos constituíram a maioria (75\%), seguidos pelos mulatos $(23,3 \%)$ e, em uma muito pequena proporção, pelos negros (1,8\%). A idade média foi de 39,81 , +- 15,61 anos. Não houve diferenças quanto à lateralidade dos cálculos, mas os homens tiveram mais cálculos bilaterais do que as mulheres.

Conclusão: Na população estudada no presente trabalho, o cálculo renal foi mais freqüente em adultos jovens, brancos e do sexo feminino, sem diferenças quanto à lateralidade. Os resultados sugerem uma possível relação entre nefrolitíase e cor da pele.

UNITERMOS: Cálculos Renais. Epidemiologia. Sexo. Lateralidade.

\section{INTRODUÇÃO}

A litíase renal é uma afecção freqüente na prática clínica. Sua presença é estimada em 3\% da população geral, podendo-se afirmar que, aos 70 anos de idade, $12 \%$ das pessoas apresentaram pelo menos um episódio de litíase. Considerando que $30 \%$ desses pacientes são hospitalizados e submetidos a alguma intervenção (extração cirúrgica, fragmentação) associa- da à redução da produtividade, pode-se avaliar o grande impacto social e econômico dessa doença ${ }^{(1,2)}$. É importante ressaltar que a litíase tem altas taxas de recorrência.

Entre o múltiplos fatores envolvidos na etiopatogenia dessa moléstia, destacam-se a supersaturação urinária, decorrente de baixa ingesta hídrica, hipercalciúria, hiperuricosúria e hipocitratúria ${ }^{(3)}$. A predisposição familiar e hereditária para a formação dos cálculos é 
conhecida $^{(4)}$. Entretanto, sua distribuição é incerta em termos de incidência, distribuição territorial e raça ${ }^{(5)}$.

Segundo a literatura, cálculos renais são duas a três vezes mais comuns em homens do que em mulheres e são incomuns em povos afro-americanos e $\operatorname{asiáticos}^{(6,7)}$. Alguns estudos evidenciaram que essa afecção, no sexo feminino, está associada ao maior número de infecções urinárias e acompanha-se de complicações mais graves e hospitalização mais prolongada ${ }^{(8,9,10)}$. Por outro lado, Parks et al.(3) afirmaram que os conhecimentos atuais ainda não são suficientes para caracterizar as diferenças da calculose renal entre homens e mulheres, já que, na maioria das publicações, as mulheres ocupam uma pequena proporção do grupo em análise ${ }^{(7)}$.

A relação entre cálculo renal e cor da pele é um dos pontos interessantes que foram pouco estuda$\operatorname{dos}^{(11)}$. A literatura mostra que a epidemiologia da nefrolitíase está relacionada à cor da pele e também a fatores genéticos e dietéticos ${ }^{(11,12)}$. Os brasileiros são uma mistura de várias raças. Existem brancos provenientes de diferentes países europeus, a maioria deles de Portugal, Itália e Alemanha. Há ainda negros, descendentes de países da África Ocidental, principalmente de Angola, Moçambique e Nigéria. Um pequeno número de índios aborígines também podem ser encontrados em diversas regiões. Mais de um terço da população brasileira é composta de uma mistura de várias raças e, por isso, é classificada como mestiça ${ }^{(11,12)}$. Essa origem mesclada de nossa população dificulta uma análise por raça. Entretanto, uma classificação de acordo com a cor da pele é possível.

Apesar da vasta experiência de determinados centros de estudo quanto à patogênese e tratamento da litíase renal, no Brasil, não encontramos estatísticas nacionais quanto a sua ocorrência. Diante desse aparente hiato, o objetivo do presente trabalho foi avaliar a nefrolitíase com relação a idade, sexo, cor da pele e lateralidade do cálculo, em pacientes tratados eletivamente de litíase renal, no Hospital das Clínicas da Universidade Federal de Minas Gerais.

\section{PACIENTES E MÉTODOS}

O presente estudo retrospectivo foi realizado com base em 400 prontuários, todos com diagnóstico estabelecido de litíase urinária, dos Serviços de Nefrologia e de Urologia do Hospital das Clínicas da Universidade Federal de Minas Gerais, no período entre 1990 e 1995. Não houve qualquer seleção desses prontuários, além do diagnóstico de nefrolitíase.
Os diagnósticos foram confirmados por dados de história clínica, associados a pelo menos um método de imagem (radiografia simples de abdome, urografia excretora, ultra-sonografia abdominal). Não foi levada em conta a composição química dos cálculos. Não foram incluídos pacientes que procuraram o Setor de Emergência do Hospital. Foram estudados pacientes que procuraram o ambulatório dos Serviços de Nefrologia e de Urologia e, também, pacientes internados eletivamente para tratamento de cálculo renal.

Os doentes foram caracterizados por sexo, idade e cor da pele (brancos, mulatos, negros). Colheram-se ainda dados referentes à lateralidade da litíase.

Foram excluídos os pacientes, quando houve qualquer dúvida relativa aos parâmetros estudados. Os resultados foram comparados por meio do teste quiquadrado. O desvio padrão foi calculado para todas as variáveis em estudo. As diferenças foram consideradas significantes para valores maiores aos correspondentes a $\mathrm{P}<0,05$.

\section{RESULTADOS}

A idade dos pacientes variou entre dois e oitenta e dois anos, com uma média de 39,81 15,61 anos. Não houve diferença entre as idades dos homens $(39,26 \pm 16,25$ anos) e mulheres $(40,16 \pm 15,04$ anos $)$.

De acordo com os registros dos prontuários, 300 pacientes eram brancos $(75 \%), 93$ pacientes mulatos $(23,3 \%)$ e apenas 07 negros $(1,8 \%)$. Não houve diferença nessa distribuição entre homens $(132-72,5 \%$ - brancos, 49 - 26,9 \% -mulatos e 1 - 0,6 \% - negro) e mulheres $(168-77,0 \%$ - brancas, $44-20,2 \%$ mulatas e $6-2,8 \%$ - negras).

A Tabela I mostra maior número de pacientes do sexo feminino com litíase urinária, apesar de a diferença com relação ao sexo masculino não ser significativa. Nessa tabela, observa-se ainda a lateralidade dos cálculos, sendo 184 à direita, 182 à esquerda e 34 bilaterais. É interessante observar que, apesar de haver mais cálculos em mulheres tanto à direita quanto à esquerda, houve maior número de homens com cálculos bilaterais.

\section{DISCUSSÃ̃O}

Os resultados do presente trabalho mostram uma freqüência maior do sexo feminino, o que contraria a literatura que afirma ser essa doença mais comum no sexo masculino ${ }^{(6)}$. Não encontramos explicação para essa particularidade em nossos achados. 


\begin{tabular}{|lcc|}
\hline $\begin{array}{l}\text { Tabela I - Relação entre sexo e } \\
\text { nefrolitíase em } \mathbf{4 0 0} \text { pacientes }\end{array}$ & localização de \\
\hline LOCALIZAÇÃO & MASCULINO & FEMININO \\
\hline Bilateral & $21^{*}$ & $13^{*}$ \\
\hline Direita & $73^{*}$ & $111^{*}$ \\
\hline Esquerda & 88 & 94 \\
\hline Total & $182(45,5 \%)$ & $218(54,5 \%)$ \\
\hline$*=P<0,05$. & \\
\hline
\end{tabular}

A análise de nossos dados mostra não existir diferença quanto à lateralidade no geral, apesar de se constar um número pouco maior de cálculos à esquerda, nos homens, e à direita, nas mulheres, porém sem significância estatística. Destaca-se, no entanto, que os homens tiveram uma freqüência de cálculos bilaterais significativamente maior do que as mulheres. Apesar de não encontrarmos subsídios na literatura, consideramos ser essa constatação importante sob aspecto de propedêutica. Talvez seja prudente uma investigação mais cuidadosa da possibilidade de litíase bilateral, quando essa afecção estiver presente no sexo masculino.

A distribuição de casos por cor da pele mostrou o predomínio dos indivíduos brancos (75\%) e uma freqüência muito baixa de negros, apenas $1,8 \%$. Não se registrou qualquer caso em paciente de origem oriental, porém a sua freqüência em nossa população é muito reduzida. Ao se compararem esses dados com os da população de Minas Gerais (brancos 57\%, mulatos $34 \%$, e negros $8 \%)^{(12)}$, verifica-se a possibilidade de existirem fatores relacionados à cor da pele que são responsáveis pela maior presença de nefrolitíase em brancos. As diferenças entre as cores não podem ser atribuídas à amostragem do hospital em estudo, já que se trata de uma instituição de referência para todas as classes sociais e seu atendimento se faz na mesma proporção de cor da encontrada na população do Estado de Minas Gerais, conforme resultado de pesquisa anterior $^{(11)}$. Esses dados estão de acordo com a literatura, que, de fato, refere menor freqüência de nefrolitíase em negros e orientais ${ }^{(6,7)} . \mathrm{O}$ presente trabalho mostra que a distribuição da nefrolitíase com relação à idade é muito ampla. Futuras investigações serão realizadas com o intuito de compreender melhor nossos achados, principalmente os referentes à cor da pele.

\section{CONCLUSÃO}

O cálculo renal, como demonstrado no presente trabalho, foi mais freqüente em brancos e sem diferença quanto à lateralidade.

\section{AGRADECIMENTOS}

Os autores agradecem ao CNPq (Conselho Nacional de Ciência e Teconologia) e à FAPEMIG (Fundação de Assistência à Pesquisa do Estado de Minas Gerais) os auxílios financeiros que permitiram a realização deste trabalho.

PETROIANU A; OLIVEIRA NETO JE \& ALBERTI LR. Epidemiology of renal calculi in a reference hospital in Belo Horizonte, Minas Gerais State. Medicina, Ribeirão Preto 34: 85-88, jan./march 2001.

ABSTRACT: Introduction: Renal lithiasis is a very common disorder in the clinical practice, and multiple factors are related to its ethiopathogenicity. However, we did not find any study about the epidemiology of lithiasis in Brazil.

Objective: To acess the influence of age, sex, skin color and laterality of stones as risk factors for renal calculi.

Methods: 400 charts of patients with diagnosis of nephrolithiasis were studied in the Nephrology and Urology Unit of the Hospital das Clínicas of Universidade Federal de Minas Gerais, in Belo Horizonte. All diagnoses were confirmed by clinical history associated to imaging studies.

Results: The presence of this disease was higher in women $(54,5 \%)$ than men. The proportion of white patients $(75 \%)$ with nephrolithiasis was significantly higher than colored $(23,3 \%)$, and black patients $(1,8 \%)$. The mean age was $39,81 \pm 15,61$ years. The side of calculi was not different in both sexes, but men presented more bilateral stones than women.

Conclusion: In the population studied in the present paper, nephrolithiasis was more frequent in white young adults, mainly in women, without prevalence of side. These results suggest possible relation between kidney stones and skin color.

UNITERMS: Kidney Calculi. Epidemiology. Sex. Laterality. 


\section{REFERÊNCIAS BIBLIOGRÁFICAS}

1 - ORTIZ V. Litíase urinária. In: PRADO FC. Atualização terapêutica, $19^{\mathrm{a}}$ ed, Artes Médicas, São Paulo, p. 1181-1182, 1999.

2 - SY FY; WONG MY \& FOO KT. Current indications for open stone surgery in Singapure. Ann Acad Med Singapure 2: 241-244, 1999.

3 - PARKS JH; COE FL \& STRAUSS AL. Calcium nephrolithiasis and medullary sponge kidney in women. N Engl J Med 306: 1088-1091, 1982.

4 - CURHAN GC; WILLETTWC; RIMMEB \& STAMPFERMJ. Family history and risk of kidney stones. J Am Soc Nephrol 10: 1568-1573, 1997.

5 - YANAGAWA M; KAWAMURA J; ONISHI T; SOGA N \& KAMEDA $\mathrm{K}$. Incidence of urolithiasis in northeast Thailand. Int J Urol 6: 537-540, 1997.

6 - HRUSKA K. Renal calculi. In: BENNETT JC, PLUM F. Textbook of Medicine, $20^{\text {th }}$ ed., W.B. Saunders, Philadelphia, p. 613-617, 1996.
7 - SERIO A \& FRAIOLI A. Epidemiology of nephrolithiasis. Nephron 1: 26-30, 1999.

8 - COE FL. Treated and untreated recurrent calcium neprolithiasis in patients with idiopatic hypercalciuria, hyperuricosuria, or no metabolic disorder. Ann Intern Med 87: 404-410, 1977.

9 - COE FL. Nephrolitiasis. Year Book, Chicago, 1978.

10 - STRAUSS AL; COE FL; DEUTSCH L \& PARKS JH. Factors that predict relapse of calcium nephrolithiasis during treatment: a prospective study. Am J Med 72: 17-24, 1982.

11 - PETROIANU A \& NETO JEO. Prevalence of acute appendicitis in a mixed population. Dig Surg 14: 195-197, 1997.

12 - INSTITUTO BRASILEIRO DE GEOGRAFIA E ESTATÍSTICA. SECRETARIA DE ESTADO DO INTERIOR DE MINAS GERAIS. SECRETARIA DE ESTADO DE PLANEJAMENTO DE MINAS GERAIS. Censo demográfico do Estado de Minas Gerais. Anuário Estatístico de Minas Gerais, p. 72, 1984.

Recebido para publicação em 06/09/2000

Aprovado para publicação em 12/02/2001 